
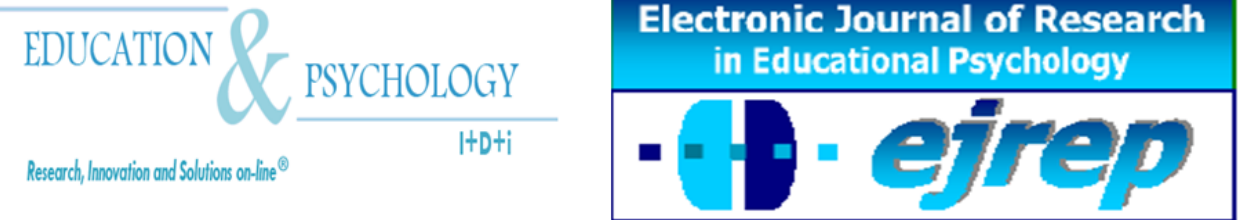

Editorial EOS

\title{
Empleo de la Geometría Dinámica como apoyo en actividades de lápiz y papel, para la comprensión de los tópicos de razón y proporción
}

Elena Fabiola Ruiz Ledesma ${ }^{1}$ y Jose Luis Lupiáñez ${ }^{2}$

${ }^{1}$ Escuela Superior de Cómputo, Instituto Politécnico Nacional, México, D.F.

${ }^{2}$ Departamento de Didáctica de la Matemática. Universidad de Granada

${ }^{1}$ México

${ }^{2}$ España

Correspondencia: Elena Fabiola Ruiz Ledesma. Av. Juan de Dios Batiz, S/N. Esq. Miguel Othon de Mendizabal. Colonia Lindavista. Delegación Gustavo A Madero. México, D.F. 07738. E-mail: elen_fruiz@yahoo.com.mx

(C) Education \& Psychology I+D+i and Editorial EOS (Spain) 


\section{Resumen}

Introducción. El presente artículo plantea la importancia de la conjunción de actividades de lápiz y papel y el uso de la tecnología con la finalidad de que los estudiantes complementen su aprendizaje de razón y proporción. Se toma como antecedente una experiencia previa sobre el uso de estrategias al resolver problemas de razón y proporción. Como respuesta al reconocimiento de los componentes cognitivos de los estudiantes se decidió tener un apoyo mediante la observación y manipulación de representaciones hechas por software de geometría dinámica.

Método. Aplicación de 3 actividades a resolver por un grupo de 29 estudiantes que cursaban sexto grado de educación primaria en una escuela pública de la Ciudad de México. Las actividades fueron resueltas con lápiz y papel y con el uso de la geometría dinámica.

Resultados. Los estudiantes construyeron figuras de forma proporcional a las dadas usando la comparación mediante la superposición de un figura en otra, a través del arrastre de estas, así como apoyándose en ampliar y reducir figuras con el arrastre de uno de los vértices de ellas. Por otra parte establecieron equivalencia de razones de forma numérica al obtener las medidas de las figuras dadas y hacer la comparación por cociente de ellas. También lograron rescatar los datos de problemas dados y los representaron mediante dibujos, después se apoyaron en ellos para dar solución a lo planteado.

Discusión y Conclusiones. En cuanto al aprendizaje que se detectó en los estudiantes, en su mayoría, lograron determinar figuras proporcionales a las dadas a través del empleo de registros de representación, como el empleo del dibujo con los datos que lograron extraer de los enunciados de los problemas, el uso de la tabla y la determinación en ella de razones tanto externas como internas, así como la relación de equivalencia entre las razones y las operaciones que emplearon para determinar valores numéricos. También mostraron un desarrollo en su pensamiento proporcional cualitativo como apoyo al pensamiento proporcional cuantitativo.

Palabras Clave: Razón, proporción, ambiente dinámico, geometría, representaciones 


\title{
Use of Dynamic Geometry as a support to paper and pencil activities for comprehension of reason and proportion topics
}

\begin{abstract}
Introduction. The present paper shows the importance of the conjunction of activities of pencil and paper and the use of the technology with the finality of the students to complete their learning about ratio and proportion. It is taken as a background a previous experience about the use of strategies when solving ratio and proportion. As an answer to the recognizing of the cognitive components of the students was decided to have a support with the observation and use of representations made by dynamic geometry.

Method. Application of three activities to solve by a group of 29 students that studied the $6^{\text {th }}$ grade in primary school in a public school in Mexico City. The activities were solved with paper and pencil and using the dynamic geometry.
\end{abstract}

Results. We observed that the students built proportional shape figures to the ones were given using the comparison by superimposing one figure over another, and also amplifying or reducing them by moving one of the figures vertex. In the other hand established ratios equivalence in numeric form when they got the given measures and made the comparison by quotient them. Also they rescued the data of the given problems and represent them with drawings, after they use them as a support to solve the problems.

Discussion and Conclusion. Most of the students could determine proportional figures to the ones given with the use representation registries that in the case of 11 years old kids are: the use of drawings putting the data that they could got from the sentences of the problems, such as the equivalence relation between the ratios and the operations that were use to get the numeric values. Also they showed a development in their proportional qualitative thinking as a support to the proportional quantitative thinking, when they could make comparisons by superimposing one figure over another using the mouse to move the figures.

Keywords: Ratio, Proportion, dynamic environment, geometry, representations.

Received: 11/10/09 Initial Acceptance: 11/23/09 Definitive Acceptance: 02/12/10 


\section{Introducción}

El problema abordado en este estudio se refiere a la importancia de la conjunción de las actividades de lápiz y papel con el uso de un programa dinámico de geometría como apoyo en la resolución de problemas de razón y proporción (simple y directa). Este estudio lo abordamos desde el análisis de una serie de tareas planteadas a un grupo de escolares mexicanos de Educación primaria, en términos de los conocimientos y las capacidades puestas en juego en su resolución.

Para llevar a cabo el estudio, en la primera parte del artículo recogemos la presencia de las nociones de proporcionalidad en el currículo de Educación primaria de México para, a continuación, destacar algunas investigaciones previas sobre el pensamiento proporcional que nos permiten disponer de herramientas conceptuales y metodológicas en nuestro trabajo. Finalmente, la primera parte se cierra con algunas reflexiones sobre las potencialidades de las nuevas tecnologías sobre geometría dinámica en el diseño de tareas sobre proporcionalidad. En la segunda parte del documento nos centramos en la descripción de los principales aspectos metodológicos del estudio empírico, así como de los principales resultados obtenidos. Nuestro principal instrumento es un modelo compuesto por varias actividades, algunas para resolver sólo con papel y lápiz y otras con el soporte de un software de geometría dinámica. Este análisis nos permite extraer una serie de conclusiones en lo cognitivo, lo conceptual y a nivel de uso del lenguaje, por ejemplo, emplearon el dibujo y lo numérico, estableciendo una conexión en ambos sistemas simbólicos, para dibujar rectángulos, por otro lado, se percibió el avance que mostraron muchos estudiantes en cuanto al reconocimiento que hay de ideas multiplicativas detrás de su pensamiento proporcional. Lo perceptual y lo intuitivo fueron un fuerte apoyo para que varios alumnos ampliaran en ambas dimensiones las figuras empleadas. La observación del dibujo original, y la ampliación realizada mediante la estrategia de duplicar su cantidad en ambas dimensiones, o triplicar u obtener la tercera, cuarta o quinta parte, además de determinar las razones implicadas en ambas figuras, muestran el tránsito que se dio de las ideas cualitativas a la cuantificación. El poder trabajar usando los tres registros de representación (el dibujo, la tabla y los datos numéricos), mostraron el avance logrado en lo conceptual. 


\section{Elementos Teóricos}

Nuestra investigación se centra en tres elementos teóricos de referencia. En primer lugar, nos interesa el tratamiento de las nociones matemáticas relacionadas con proporcionalidad en las directrices curriculares. En segundo lugar, nos centramos en diferentes investigaciones relacionadas con el pensamiento proporcional y, finalmente, nos preocupamos del papel que pueden jugar las nuevas tecnologías en el aprendizaje de esas nociones matemáticas.

\section{Proporcionalidad y curriculum}

Muchas de las necesidades que presentan los alumnos de educación secundaria o Bachillerato, tienen sus raíces en la escuela elemental. Al respecto, Guerrero, Gil y Blanco (2006), señalan que el aprendizaje depende tanto de la intervención del estudiante como del profesor. En efecto, hay temas de matemáticas que se introducen en Educación primaria y la forma como se traten, así como la construcción que de los conceptos involucrados en ellos se haga, es lo que permite a los estudiantes lograr la comprensión y la aplicación de conceptos que abordarán en los siguientes niveles educativos. Este es el caso del tema de razón y proporción, ya que la enseñanza de estos tópicos se inicia en la escuela elemental (SEP, 2001 ${ }^{\mathrm{a}}$ y 2001b) y sirven de base para posteriores conceptos. Lesh, Post y Behr (1988), señalan que muchos conocimientos críticos consisten fundamentalmente en el reconocimiento de patrones similares o semejanza estructural en dos diferentes situaciones.

Debido a que el razonamiento proporcional concuerda con una de las formas más comunes de semejanza estructural, frecuentemente se relaciona con algunos de los más importantes, elementales, pero profundos conceptos en el nivel básico de muchas áreas de las matemáticas. De esta forma, el razonamiento proporcional es relacionado con alguno de los primeros "problemas" conceptuales en el currículum: equivalencia de fracciones, división larga, ubicación de valor y porcentaje, conversión de medidas y razones de cambio.

En las clasificaciones anteriores de áreas temáticas, como la temática de fracciones, la de porcentaje, la de conversión de medidas, entre otras, hay distintos tipos genéricos de problemas relacionados con proporciones y que emergen de manera natural, como es el caso de los problemas de valor desconocido o los problemas de comparación, así como los problemas 
de selección para lo cual el estudiante puede identificar cuándo una figura está reducida o amplificada a la figura proporcionada.

Lesh, Post y Behr (1988) señalan también que toda persona que resuelve un problema referido a proporciones, no necesariamente usa el razonamiento proporcional. De hecho, uno puede notar una simple relación numérica (si $A$ es tres veces $B, X$ debe ser tres veces $D$ ) o puede usar un algoritmo mecánico tal como una multiplicación cruzada. Por otro lado, estos mismos autores, destacan que el razonamiento proporcional abarca una amplia y compleja gama de habilidades cognoscitivas que incluyen tanto la dimensión matemática como la psicológica.

\section{Estudios relacionados con el pensamiento proporcional}

Para Piaget e Inhelder (1978), la noción de proporción empieza siempre de una forma cualitativa y lógica, antes de estructurarse cuantitativamente. Piaget (1978), señala que el niño adquiere la identidad cualitativa antes que la conservación cuantitativa y hace una distinción entre comparaciones cualitativas y la verdadera cuantificación.

Este autor sostiene que entre los 11 y los 12 años, el sujeto toma consciencia de la presencia de la noción de proporción en diferentes ámbitos, tales como las proporciones espaciales (figuras semejantes), las relaciones entre pesos y longitudes de los brazos en la balanza o las probabilidades. En el caso de la balanza de barra, por ejemplo, el sujeto puede comprender, mediante la manipulación del dispositivo, que es posible conservar el equilibrio teniendo dos pesos iguales a las mismas distancias del centro, pero también se conserva el equilibrio disminuyendo un peso, pero alejándolo y aumentando el otro, aunque aproximándolo al centro. La comprensión de esta proporcionalidad (tanto directa como inversa), se da en primer lugar por vía cualitativa: "es lo mismo aumentar el peso que la distancia", para presentarse después en formas métricas simples: "disminuir el peso aumentando la longitud equivale a aumentar el peso disminuyendo la longitud".

Otros estudios de investigación se han enfocado en las necesidades de la instrucción. Por ejemplo, Hart (1988), señala que el pensamiento proporcional está presente en el adolecente y que evoluciona una vez que éste ha construido ciertos conceptos. Para Hart, algunos 
niveles de generalización, tales como los manejos de razones o maneras de generar equivalencias, ocurren cuando se usan estrategias multiplicativas.

Además, Freudenthal (1983) y Streefland (1984, 1990, 1991 y 1993), combinaron aspectos didácticos con la reflexión matemática acerca de razón y proporción. Los reportes de estos investigadores nos permiten contemplar sus contribuciones a estos campos. Basado en estudios citados, fue posible crear las actividades en lápiz y papel que se proponen, debido a que Freudenthal hace énfasis al trabajo de las razones tanto externas como internas, así como a la comparación de figuras mediante la superposición, que para este investigador es la idea de medida. De esta forma y tomando como referencia lo mencionado es como se crearon los diseños de lápiz y papel pues en el de la cancha de fútbol se requiere hacer establecer ya sea razones internas o externas para resolverla. En cuanto a Streefland (1984, 1990, 1991 y 1993) él enfatiza el empleo de recursos didácticos para la comprensión de los tópicos de razón y proproción, así como el que a temprana edad se ve en el estudiante un tratamiento de la proporción mediante ideas intuitivas. Además, apoya la idea de cambio en perspectiva lo que tomamos como soporte para poder llevar el trabajo de lápiz y papel al uso de un programa dinámico asistido por una computadora, una instrucción diseñada bajo acercamiento constructivista.

\section{La contribución de nuevas tecnologías}

El incremento de proyectos educativos que incluyen el uso de un componente tecnológico está constatado en la investigación actual (English, 2009). Esta situación motiva a los maestros e investigadores a incluir nuevos aparatos tecnológicos en sus actividades de enseñanza. Por lo tanto, la traducción de nuestra reflexión al rol que estos aparatos pueden jugar en la enseñanza de nociones tales como razón y proporción es inmediata, tal y como lo señalan Lupiáñez y Moreno (2001).

En este documento, introducimos algunas actividades en el ambiente de geometría dinámica Cabri-Géomètre ${ }^{1}$. El diseño de estas actividades se basó en las observaciones y en los resultados obtenidos del estudio reportado por Ruiz y Lupiañez (2009), sobre los obstáculos psicopedagógicos detectados al aplicar un cuestionario a estudiantes de Educación prima-

\footnotetext{
${ }^{1}$ www.cabri.com
} 
ria en la ciudad de México, así como en el trabajo de Ruiz (2002), Ruiz y Valdemoros, (2002 y 2004), mediante un programa didáctico basado en tareas que se resuelven con lápiz y papel.

Ya desde su diseño, el paquete Cabria-Géomètre proporciona un entorno de Geometría que cuenta como característica primordial la función de arrastre: al arrastrar alguno de los elementos que conforman una figura, es posible generar figuras proporcionales. Esta característica es importante en las actividades didácticas que se han diseñado previamente en lápiz y papel (Santos y Espinosa, 2002). Como destaca Streefland, (1991), el cambio de un contexto de resolución en lápiz y papel a una resolución en computadora, es un cambio en perspectiva. Es decir, se crea un modelo y se trata de explotar al máximo desde una idea y, posteriormente, se retoma dicho modelo explotándose a la luz de otra idea. El cambio en perspectiva se caracteriza por el intercambio de una parte de la información en la situación problema de la que se está preguntando.

La utilización de la conservación de la proporción en la construcción de micro mundos puede ser usada en el desarrollo de las actividades didácticas, al menos con tres posibles enfoques: i) como verificador de los cálculos que hagan los alumnos; ii) como generados de nuevos problemas por medio del arrastre de algún punto libre conveniente; iii) como ilustrador de la transición entre las configuraciones posibles. Desde esta óptica, Cabri-Géomètre se convierte en un recurso para favorecer el reconocimiento y desarrollo de patrones perceptuales; por ello, algunas de las construcciones que se hagan tendrán como propósito dar una mayor fidelidad conceptual y fidelidad epistémica. Más adelante se describe brevemente cómo pueden realizarse estas construcciones en este entorno de geometría dinámica.

\section{Planteamiento del problema y objetivos}

En trabajos previos, se ha constatado que al enfrentar tareas de razón y proporción, los estudiantes de sexto grado de primaria presentan varias dificultades (Ruiz, 2002), por lo que se proponen actividades en dos ambientes, lápiz y papel y un ambiente de geometría dinámica para apoyar a los estudiantes a superar estas dificultades. De esta manera, es posible acotar los dos objetivos propuestos de esta investigación: 
- Revisar las estrategias que emplean los estudiantes de un grupo de sexto grado de primaria al resolver actividades en lápiz y papel sobre razón y proporción.

- Revisar los beneficios que aporta el uso de un programa dinámico al resolver las mismas actividades de razón y proporción en lápiz y papel por un grupo de estudiantes de sexto grado de primaria.

\section{Método}

\section{Participantes}

Para el estudio empírico de nuestra investigación, se trabajó con un grupo de 29 estudiantes de sexto grado de Educación primaria, niños de 11 años de edad, de una escuela pública de la Ciudad de México.

\section{Instrumentos}

Se diseñaron y llevaron a la práctica dos programas didácticos sobre razón y proporción, uno de ellos centrado en actividades para realizar con papel y lápiz y otro estructurado en torno a actividades en el ambiente Cabri-Géomètre.

Diseño del Programa didáctico desarrollado con lápiz y papel con estudiantes de educación primaria.

El programa didáctico del experimento educativo estuvo conformado por tres actividades de enseñanza de las nueve empleadas por Ruiz (2002), que fueron experimentadas en diversos momentos, según lo requería la implementación del desarrollo del estudio de investigación. Estas tres actividades fueron elegidas porque permitían, de una forma más accesible para el alumno, reproducir las figuras en el entorno informático para su resolución.

En la Tabla 1 se muestran los títulos de las tres actividades que se utilizaron, los propósitos que se persiguen, el seguimiento de los contenidos y la forma en que se pretendió que fueran trabajados con los estudiantes. 
Tabla 1. Organización de la propuesta de Enseñanza

\begin{tabular}{|c|c|c|c|}
\hline Modelo & Propósito(s) & $\begin{array}{c}\text { Lo que se incorpora en cada } \\
\text { sesión }\end{array}$ & $\begin{array}{c}\text { Estructura de la } \\
\text { dinámica del } \\
\text { trabajo }\end{array}$ \\
\hline $\begin{array}{l}\text { Torneo de } \\
\text { fútbol }\end{array}$ & $\begin{array}{l}\text { Usar diferentes razones al tra- } \\
\text { bajar la proporción. }\end{array}$ & $\begin{array}{l}\text { Determinar las razones en las que } \\
\text { se encuentran diferentes medidas } \\
\text { de tres canchas de fútbol. }\end{array}$ & $\begin{array}{l}\text { Grupal-Colectivo- } \\
\text { Individual. }\end{array}$ \\
\hline $\begin{array}{l}\text { Construye tu } \\
\text { propia cancha }\end{array}$ & $\begin{array}{l}\text { Usar diferentes modos de re- } \\
\text { presentación }\end{array}$ & $\begin{array}{l}\text { Pintar en el patio de su escuela la } \\
\text { mayor cancha posible y que sea } \\
\text { proporcional a la dada. }\end{array}$ & $\begin{array}{l}\text { Colectivo- } \\
\text { Individual. }\end{array}$ \\
\hline $\begin{array}{l}\text { La fotografía } \\
\text { de tu equipo. }\end{array}$ & $\begin{array}{l}\text { Trabajar con razones decima- } \\
\text { les, si llegara a darse el caso, al } \\
\text { determinar proporciones. }\end{array}$ & $\begin{array}{l}\text { Rescatar los procedimientos que } \\
\text { usa el estudiante al trabajar pro- } \\
\text { porciones. }\end{array}$ & $\begin{array}{l}\text { Grupal-Colectivo- } \\
\text { Individual. }\end{array}$ \\
\hline
\end{tabular}

\section{Procedimiento}

En las tres actividades trabajadas, se le dio especial atención a los diferentes modos de representación que los estudiantes usaron cuando estuvieron confundidos con las situaciones propuestas. Intentamos que los estudiantes pudieran usar indistintamente uno de los siguientes tres registros de representación: el dibujo, la tabla o los datos numéricos.

Es importante destacar que, como señala Franco (2008), una vez que el niño percibe claramente la realidad él puede actuar, pues para que el estudiante logre emplear los registros de representación requiere haber percibido con claridad la situación planteada.

A continuación se muestran las actividades de los tres modelos de actividades de enseñanza que fueron desarrollados con un grupo de sexto grado de Educación primaria, primero empleando lápiz y papel y después haciendo uso del programa dinámico de geometría.

\section{Actividades realizadas con papel y lápiz}

\section{Actividad 1: Torneo de Fútbol}

El propósito de esta actividad era que los alumnos usasen diferentes razones, al trabajar la proporción; primero de forma intuitiva al comparar y determinar si cabe dos veces o tres veces, y luego de forma explícita al emplear cantidades. Para ello, trabajaron de manera indi- 
vidual al completar los datos que se les solicitaban, extrayéndolos del dibujo de la cancha oficial. Una vez conocidos los valores de las distintas dimensiones de ésta, determinaron aquéllos requeridos para la cancha "B" que es donde jugarían en el torneo los alumnos de $5^{\circ}$ y $6^{\circ}$ grado, así como los valores de las medidas de la cancha "C" que se asignaría para los niños pequeños. En las figuras 1, 2 y 3 se muestran los dibujos de las canchas:

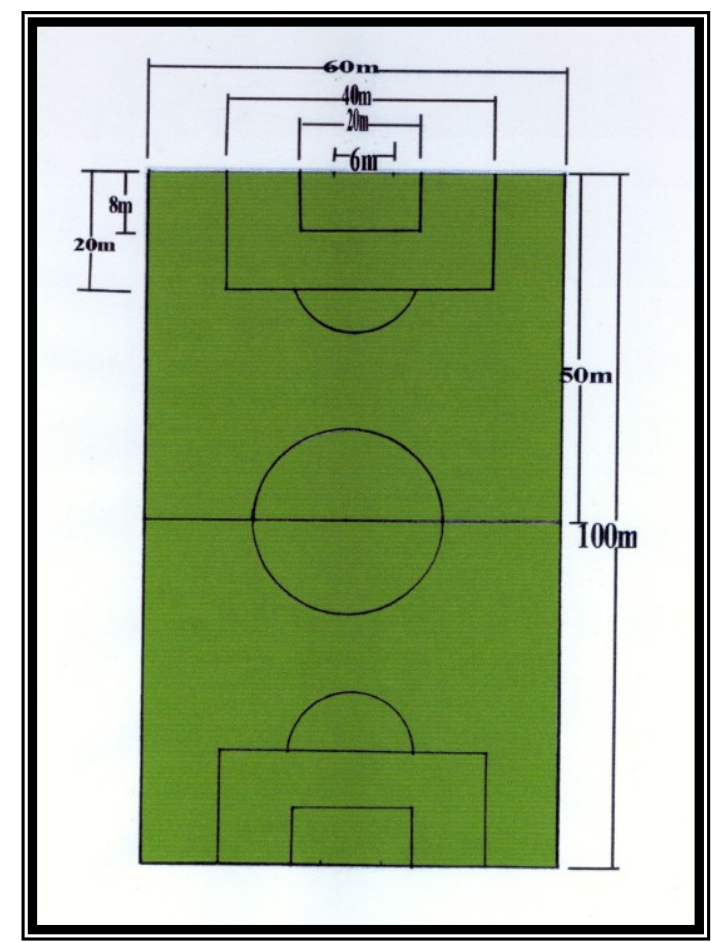

Figura 1. Cancha "A" (con las medidas oficiales).

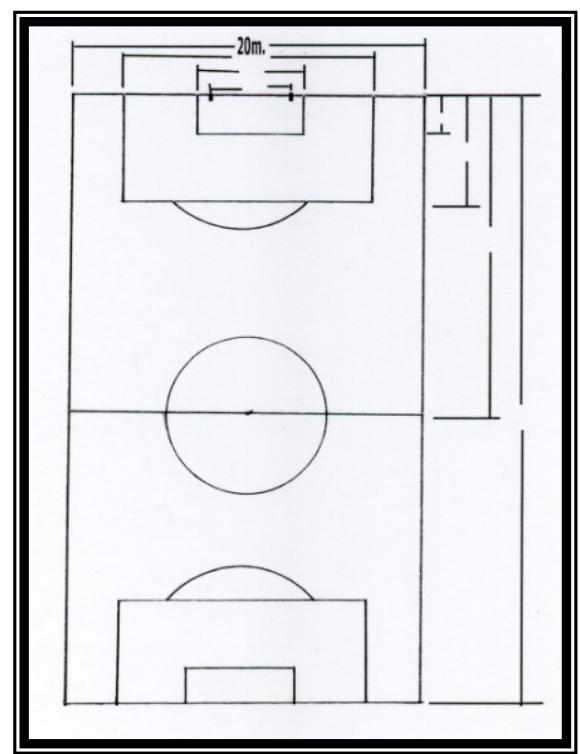

Figura 2. Cancha B

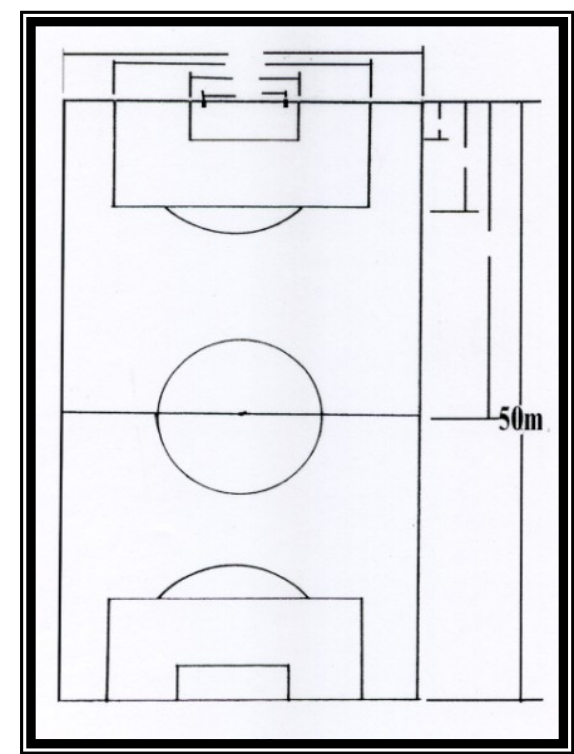

Figura 3. Cancha C 
Actividad 2: Construye tu propia Cancha

La finalidad en este caso era que los alumnos trabajasen un problema en donde utilizasen la razón para determinar las medidas, así como que usaran diferentes registros de representación al trabajar la proporción.

En principio los estudiantes trabajaron por equipo, leyeron las indicaciones en la hoja dada, las cuales son las siguientes: "Las medidas del patio de la escuela son 40 metros de largo por 12 metros de ancho. Vas a construir la mayor cancha de fútbol que se pueda, sin pasarte de las medidas dadas y que sea proporcional a la cancha oficial."

Actividad 3: La fotografía de tu equipo

En esta tercera actividad, el propósito era que los alumnos emplearan proporciones y razones, apoyándose en operadores naturales y que trabajasen en los tres registros de representación: el del dibujo, el de la tabla y las operaciones numéricas.

A cada equipo se le entregó su hoja de trabajo incluyendo la fotografía de uno de los equipos. Después de leer las reglas del juego, se comentó su contenido de manera colectiva, para saber si los alumnos habían entendido lo que se les estaba demandando.

A los alumnos se les indicó que podían emplear dibujos, tablas, hacer operaciones, o lo que ellos consideraran que les permitiría determinar las estaturas de las personas que estaban en la foto. La hoja de trabajo se muestra en la figura 4

Actividades de proporcionalidad en Cabri-Géomètre

Los estudiantes en los últimos grados de Educación primaria tienen potencial para la adquisición y manejo de las nociones de razón y proporción, en un contexto coherente con su previo conocimiento matemático. Esta potencialidad se demostró claramente en la luz de los resultados obtenidos a través del desarrollo de la propuesta de enseñanza de Ruiz (2002). La actual propuesta provee ajustes para las actividades descritas en los próximos párrafos y el objetivo es que los estudiantes complementen su aprendizaje con la observación y manipulación de representaciones hechas por la geometría dinámica en el ambiente de Cabri-Géomètre. 
En cualquier caso, algunas de las tareas están mejor adaptadas para la continuación del trabajo al principio de la Educación secundaria, no para los niveles de Educación primaria (grados 16). Suponemos que los estudiantes en ambos niveles de educación han adquirido las habilidades básicas para manejar este software.

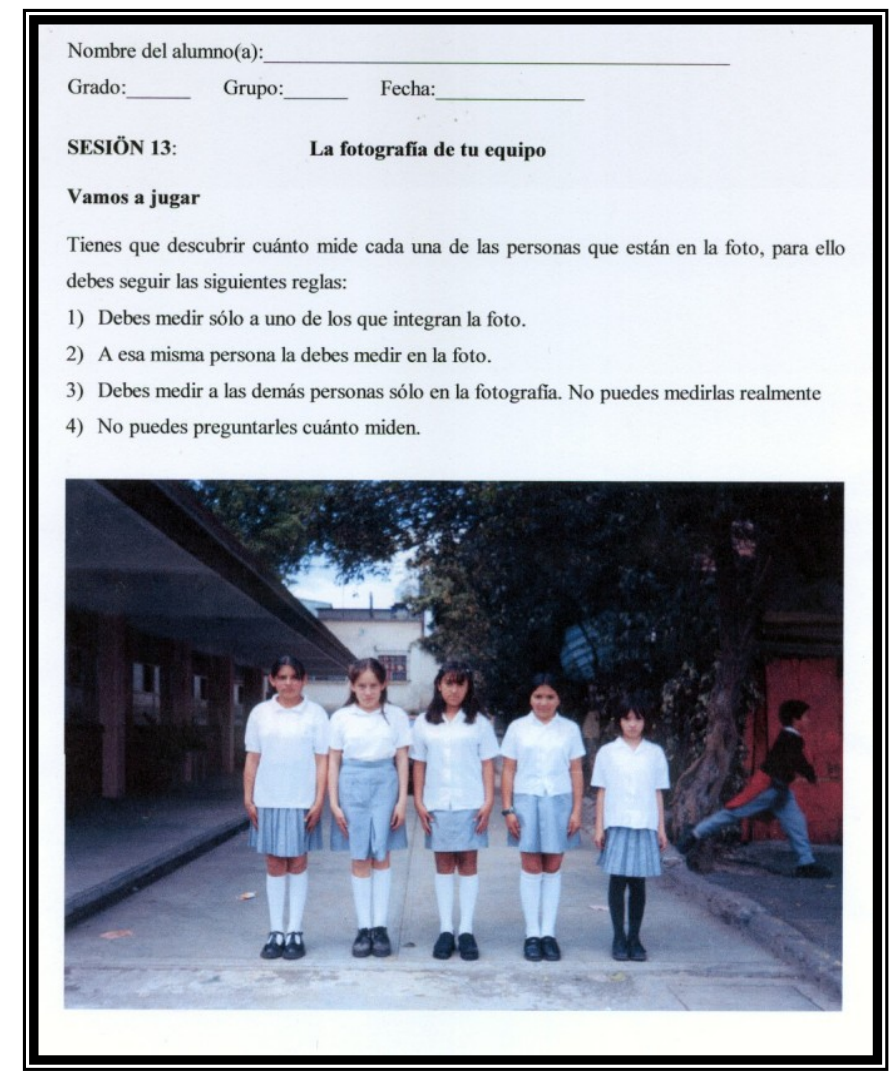

Figura 4. Actividad. La fotografía de tu equipo

\section{(Actividad $\left.1^{a}\right):$ Reconocimiento de patrones}

Se empleó esta actividad para poder familiarizar a los estudiantes con el programa de geometría dinámica y el reconocimiento de figuras proporcionales. Asimismo para que los escolares descubrieran una de las bondades del programa que es el arrastrar figuras y sobreponerlas, acciones que habían realizado con lápiz y papel, o el de arrastrar un vértice de una figura y agrandarla o reducirla, que en ambos casos el programa las hace proporcionales a la original. Esto va acorde a lo señalado por Freudenthal, (1983), Piaget (1978) y Streefland $(1984,1990,1993)$. 
El objetivo es que los estudiantes arreglen los rectángulos de acuerdo a su visual percepción, y una vez hecho eso, pueden mostrarse las medidas de los rectángulos. Entonces los estudiantes pueden determinar las razones en las que se encuentran los lados de los rectángulos al determinar las veces que cabe un lado en otro.

(Actividad 1b): Construcción de figuras proporcionales y no proporcionales.

El propósito de esta actividad es introducir un criterio para la construcción de rectángulos proporcionales entre sí. Los rectángulos deben ser construidos de manera que compartan un vértice y sus diagonales a través de la misma línea recta.

En los primeros cursos de Educación secundaria, es posible introducir la invariación de semejanza de las figuras usando la noción de homotecia. Este tema se convierte especialmente fácil y al mismo tiempo muy ilustrativo en el ambiente de Cabri-Géomètre; es muy interesante ver como el punto móvil $P$ genera familias de figuras similares.

\section{Actividad 2: Construcción de una cancha de fútbol}

Recordando las dimensiones más sobresalientes de una cancha de fútbol real, tenemos lo siguiente: la proporción en la que se encuentra el largo al ancho de la cancha es de 60 a 100, es decir 3 a 5; la razón del largo del área grande al largo del campo es de 20 a 100, es decir 1 a 5; el largo del área chica al largo del campo dan una razón de 8 es a 100, ó 2 a 25; el ancho del área grande al ancho del campo da una razón de 40 a 60, es decir 2 a 3; el ancho del área chica al ancho del campo 20 a 60, dicho de otra forma 1 a 3; el ancho de la portería al ancho del campo, 6 a 60 equivalentemente 1 a 10. Además, hay una línea a mitad del campo. Todas estas proporciones se reflejan fielmente en el modelo.

Existen otras dimensiones características en el campo, como la distancia del centro de la portería al punto de penalti. Se reflejaron selectivamente algunas de ellas para dar una aproximación a la apariencia del campo. 
Actividad 3: La fotografía de tu equipo

La medición de segmentos y la razón entre ellos puede ser obtenida por los comandos convenientes del Cabri-Géometrè; la precisión de estos valores puede ser ajustada con 1, 2, 3, 4 o más decimales, cambiando el parámetro en el menú de preferencias. Los valores obtenidos se actualizan al manipular los puntos libres de la construcción (ver figura 5).

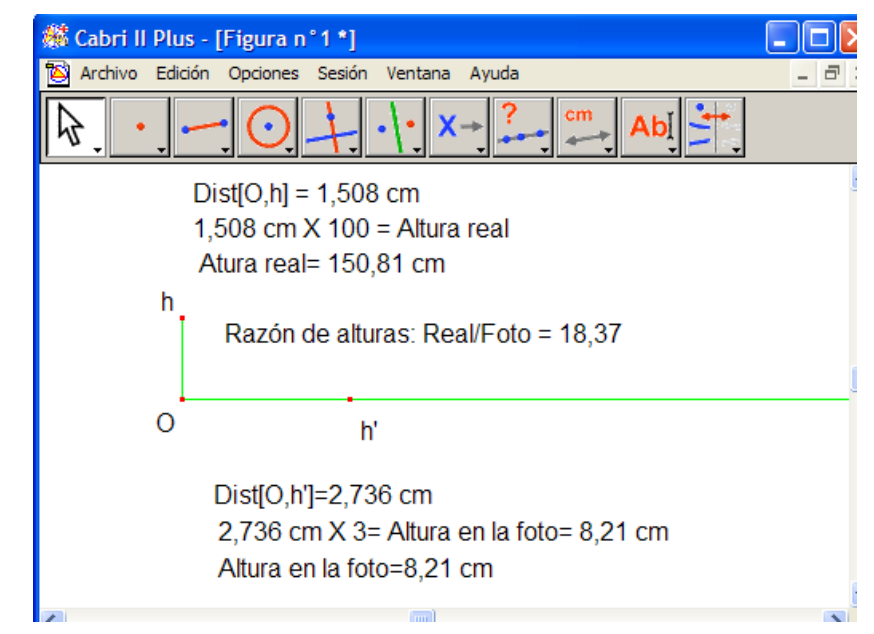

Figura 5. Construcción de segmentos que representan la estatura real y la de la foto

La construcción tiene la característica de que al manipular el punto $h$, la longitud del segmento $O h^{\prime}$ se ajusta para preservar la razón entre altura real y altura en la foto; y al manipular $h$ 'se cambia la razón entre dichas medidas.

\section{Resultados}

Este apartado se divide en dos, en el apartado I se presentan los resultados de las tres actividades del programa didáctico llevado a cabo en lápiz y papel y en el apartado II se muestran los resultados del programa con cabri-geometre. A su vez, cada apartado tiene tres incisos a, b y c que contienen los resultados y análisis de cada actividad. 
Apartado I Resultados obtenidos en el programa con lápiz y papel

\section{Actividad 1: Torneo de Fútbol}

Para determinar las medidas de la cancha "B", los alumnos se fijaron en que el largo de ella era la mitad del de la cancha oficial y como se les indicaba que tenía que ser proporcional, comentaron que en todas sus dimensiones lineales la cancha "B" debía medir la mitad con relación a la otra cancha. Para obtener la mitad dividieron entre dos.

\section{a) Análisis de la Actividad 1}

Muy pocos estudiantes (10.24\%) obtuvieron la mitad restando una cantidad fija a la dada y comprobaron, sumando entre sí el mismo valor. El trabajar con la mitad no permitía reconocer si estos tres alumnos estaban pensando en la multiplicación como una suma abreviada o si utilizaban a la adición de manera incorrecta frente a una tarea de proporcionalidad. Es decir, no se sabía con seguridad si se estaba frente a un problema de no reconocer a la multiplicación como la antesala de la proporción. Los restantes estudiantes (89.6\%) obtuvieron la mitad mediante la operación de dividir entre dos.

Fue necesario realizar algunos ejercicios que les permitieran a los niños percibir que el sumar dos veces una cantidad es como multiplicarla por dos y que podían sumar tantas veces una misma cantidad, correspondiendo ese número de veces al factor por el cual multiplican la cantidad. Esto es, concluyeron que una multiplicación es una suma abreviada; así, sumar tres veces un número es igual a multiplicarlo por tres, pero no es igual que sumarle el número tres a una cantidad para obtener el triple de su valor. Se hicieron varios ejercicios al respecto.

Con relación a la cancha "C", algunos estudiantes se dieron cuenta de que una de las medidas dadas correspondía a la cuarta parte de la medida homóloga en la cancha oficial. Dijeron que "como debía de ser proporcional, todas las dimensiones lineales deberían de ser la cuarta parte de la oficial" y determinaron las medidas dividiendo entre cuatro. (55.2\% de los9 alumnos realizaron este procedimiento). El resto de los escolares correspondiente al $44.8 \%$ se percataron que una de las medidas de la Cancha "C" correspondía a la mitad de la medida homóloga de la cancha "B" y sacando la mitad de los demás valores, obtuvieron los valores restantes para determinar que fuera proporcional. 
Cuando se solicitó que algunos estudiantes dieran sus resultados, se llegó a concluir que la mitad de la mitad de un valor, es la cuarta parte de éste.

Se observó que varios alumnos (65.5\%), elaboraron una tabla para vaciar los datos de las canchas "C", "B" y después leyeron los valores de aquélla para mencionar las razones que se les solicitaban. Otros niños (34.5\%), leyeron las cantidades extrayéndolos directamente de los dibujos de las canchas. En esta ocasión, la notación que emplearon para escribir las razones fue fraccionaria. Con ello, se puede notar que algunos estudiantes empezaron a usar y relacionar distintos registros de representación para expresar a las razones: el del dibujo, el de la tabla y el de las operaciones numéricas.

\section{Actividad 2: Tornéo de Fútbol}

En general, se observó que todos los equipos emplearon el dibujo tanto para representar el patio de su escuela como la cancha oficial, donde anotaron las medidas tanto de largo como de ancho. El siguiente paso (efectuado por algunos alumnos) fue obtener la mitad de lo que medía tanto el largo como el ancho de la cancha oficial y revisar si cabían en las medidas de su patio y así continuaron, obteniendo mitades. Acción que se observa es común en los alumnos, según Piaget (1978) y Piaget e Inhelder (1978).

\section{b) Análisis de la Actividad 2}

Se detectó que tres estudiantes sólo pusieron atención en una de las dimensiones, al obtener mitades y si esa era menor a la correspondiente de la cancha oficial, inmediatamente decían que ya habían encontrado lo que se les pedía.

Pocos estudiantes $(13.8 \%)$ mostraron otra de las acciones señaladas en ese estudio previo, que consistía en que los alumnos se centraban en una sola dimensión al dibujar una figura proporcional a otra. La mayoría de los estudiantes del estudio que se presenta en este documento, lograron establecer que si una medida lineal se reduce a la mitad, todas las demás también deben reducirse para que sea proporcional a la original.

El resto de los estudiantes equivalente al $86.2 \%$, obtuvieron mitades en ambas dimensiones. 
Se encontró en pocos alumnos (24.1\%), otra dificultad, la cual consistía en que sólo consideraban una de las medidas para ver si era menor a la correspondiente en el patio de su escuela, y de ser así, los alumnos decían que "esa cancha cabía en él”. Pero cuando se les preguntaba qué pasaba con la otra dimensión, comentaban que "ésta era más grande que la del patio”, entonces tenían que seguir reduciendo, pero fijándose que tanto el largo como el ancho de la cancha fueran más pequeños que los correspondientes al patio de su escuela. Así, sacando mitades, obtuvieron una cancha proporcional pero se dieron cuenta que "no era la más grande que podía caber en el patio de la escuela".

Después de varios ensayos, esto es, después de seguir el proceso de obtener mitades, en su totalidad, los 29 estudiantes decidieron sacar la tercera parte de las medidas así como la cuarta parte. Finalmente, llegaron a obtener la quinta parte de las dimensiones de la cancha oficial y dijeron "encontramos una cancha que es proporcional y al mismo tiempo es la más grande que cabe en el patio de la escuela” (ver Figura 6).

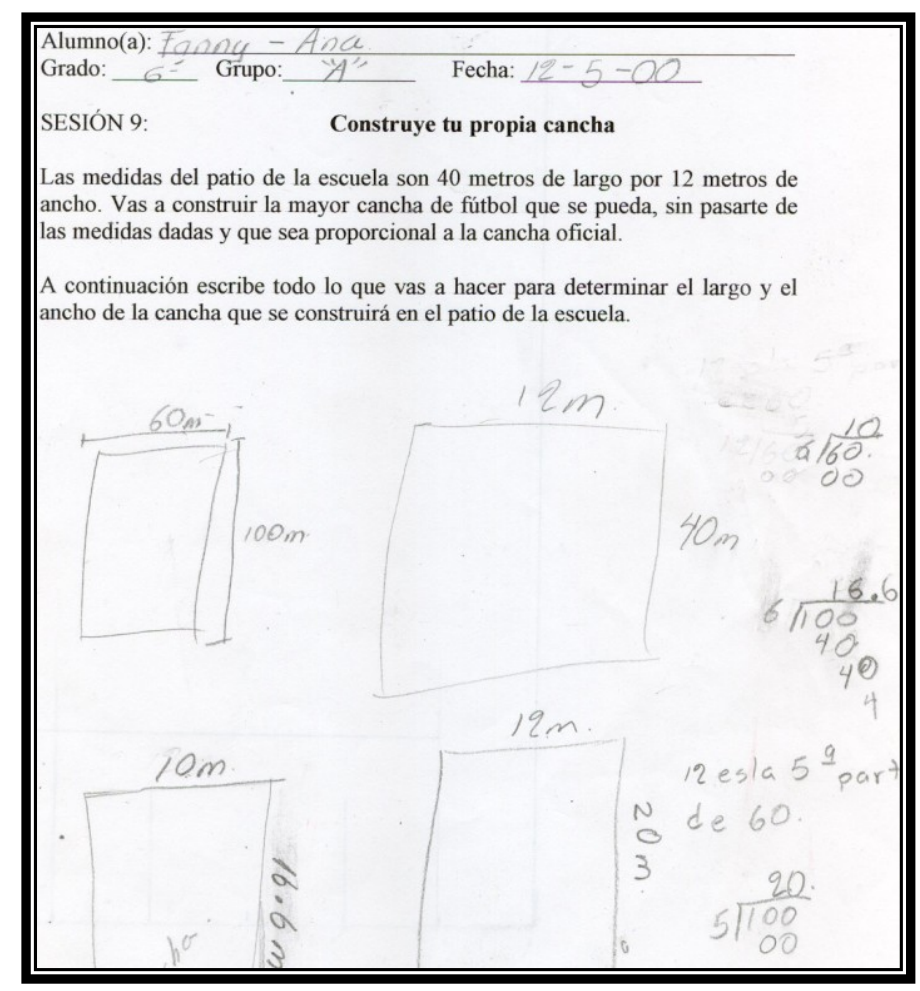

Figura 6. Trabajo en equipo 
Los alumnos trabajaron con números naturales, aunque, al efectuar divisiones y encontrar números decimales, optaban por buscar que las dimensiones de la cancha que construirían en su patio correspondieran a números naturales. Es fácil observar que se trata de un problema de optimización para el cálculo de máximos, que podía ser trabajado en un nivel superior de educación, conocida la función y la herramienta del cálculo correspondiente. Pero en este nivel, fue trabajado desde la aritmética, empleando el dibujo y la tabla como registros de representación.

\section{Actividad 3: La fotografía de tu equipo}

La mayoría de los equipos midieron en la vida real a una persona integrante de la foto y también obtuvieron su medida en la fotografía. Después obtuvieron la medida de las demás personas en la fotografía y se formularon la pregunta “¿cuánto mide cada una de ellas en la vida real, conocidos los datos mencionados?"

\section{c) Análisis de la Actividad 3}

La mayoría de los estudiantes, que en porcentaje equivale al 89.6\%, representó con dibujos las estaturas reales y las de la foto, transformando a milímetros los valores obtenidos en centímetros.

Los alumnos se dieron cuenta que estaban buscando una figura que fuera proporcional para lo cual establecieron una razón externa, ya que relacionaron medidas de diferente escala (medida de la estatura real con medida de la estatura en la foto). Escribieron la fracción estatura real 1/estatura en la foto1 (mostrada en la figura 8) y al efectuar la división entre ambos valores obtuvieron una cantidad decimal. Hubo un momento en que estos alumnos se detuvieron y no sabían qué hacer con el valor obtenido; es decir, se notó cierto desconcierto para proseguir resolviendo la tarea, por lo que se les pidió que repitieran en voz alta lo que hasta el momento habían realizado y qué es lo que pretendían con las dos figuras dibujadas. Los alumnos respondieron "que sean proporcionales". Al preguntarles si recordaban lo que significaba que dos figuras fueran proporcionales, los niños mencionaron que sí y que en este caso deberían de obtener la misma razón entre la estatura real de otra persona que aparece en la foto con respecto a lo que medía en ella. 
A continuación efectuaron operaciones y al percatarse que el cociente de la división entre la estatura real y la de la foto debía de ser el mismo en los dos casos (persona 1 y persona 2), y que tenían que descubrir el valor de la talla de la persona en la vida real, comentaron que en la división conocían dos valores, el que representa al divisor y al cociente, por lo que multiplicando ambos encontrarían el dividendo, que representaba la medida buscada. Ese fue su método de resolución de la tarea. Después de realizar sus multiplicaciones con la razón obtenida y el valor de cada estatura en la foto, llenaron una tabla.

Se destaca que emplearon los tres registros de representación para resolver el problema y pudieron hacer el tránsito de uno a otro.

\section{Apartado II Resultados del Programa empleando la geometría dinámica}

\section{Actividad 1a: Reconocimiento de patrones}

En esta primera actividad, se les mostró a los estudiantes una familia de rectángulos. Estos rectángulos fueron movidos y superpuestos sin deformación en la pantalla. El sobreponer un rectángulo en otro es una forma de medir, tal y como lo señala Freudenthal (1983).

\section{a) Análisis de la Actividad 1}

Se les pidió a los estudiantes elegir dos rectángulos que fueran proporcionales a simple vista. Una vez que los estudiantes sobrepusieron un rectángulo en otro pudieron arrastrar un punto extremo del rectángulo superpuesto para hacerlo más grande y revisar si al agrandarlo quedaba del tamaño del rectángulo que se encontraba abajo. De esta forma, los 29 estudiantes verificaron que el rectángulo seleccionado era proporcional o no al dado. Esta actividad es claramente relacionada a la semejanza de figuras, como señalan Lesh, Post y Behr (1988), aunque el término de semejanza no es usado.

Después de discutir si había una relación en casos simples, tal cual una relación puede generalizar todos los casos, surgen las primeras medidas y la computación de razones. El objetivo de esta actividad era que los estudiantes aprendan las herramientas básicas del software Cabri-Géomètre y las usen para trabajar con razones. Para este propósito, los 29 estu- 
diantes dibujaron segmentos lineales y después los midieron para descubrir las razones entre sus dimensiones (ver Figura 7).

Empezando con estas simples manipulaciones, se puso de manifiesto capacidades importantes de los alumnos: tomar medidas, manipular medidas y las posibilidades de computación.

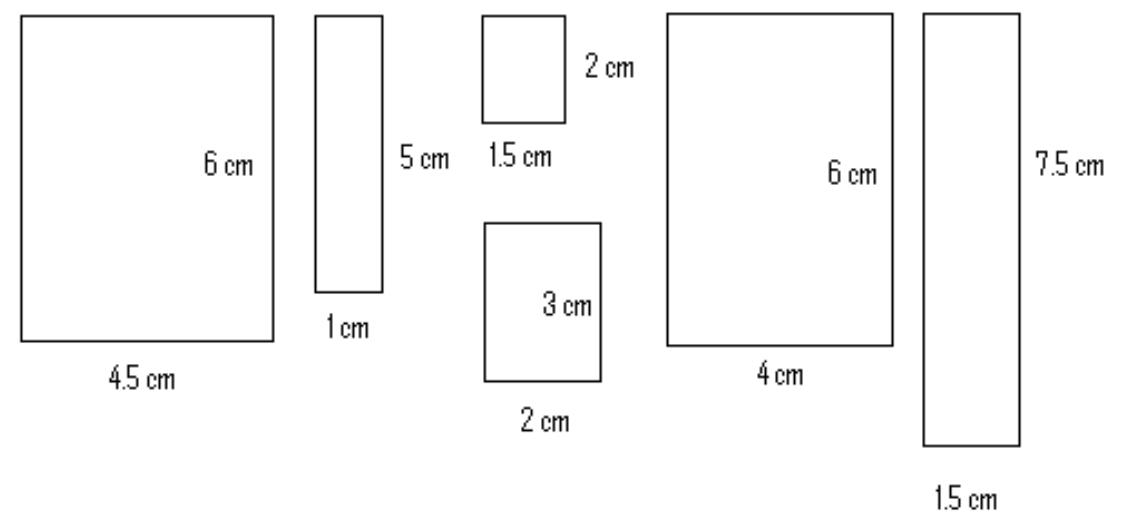

Figura 7. Actividad 1 en entorno dinámico

b) Análisis de la Actividad $1 b$

Los rectángulos que construyeron los estudiantes, primeramente fueron construidos de manera que compartían un vértice y sus diagonales a través de la misma línea recta (Figuras 8 y 9). Los 29 estudiantes movieron el vértice $\mathrm{F}$ en línea recta y mantuvieron un registro de las diferentes medidas en la tabla, podemos verificar que la proporción resultante no cambia, con lo que se obtuvo una familia completa de rectángulos similares. Igualmente, en la Figura 12 podemos comprobar que los alumnos añadieron la misma constante a las dimensiones de los lados del rectángulo, y en este caso no obtuvieron un rectángulo proporcional al original. Después de desarrollar esta tarea, fue posible confrontar la actividad "torneo de fútbol" implementando este mismo criterio. 


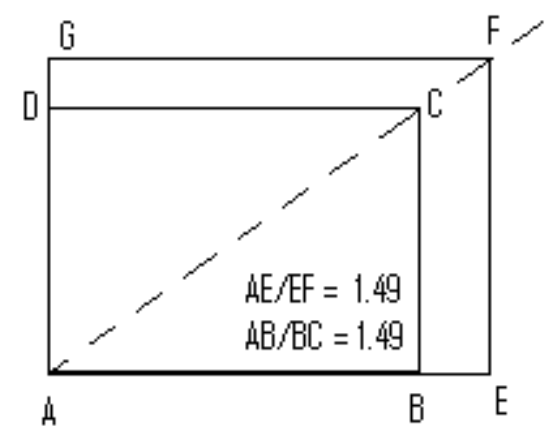

Figura 8. Construcción de rectángulo proporcional en entorno dinámico

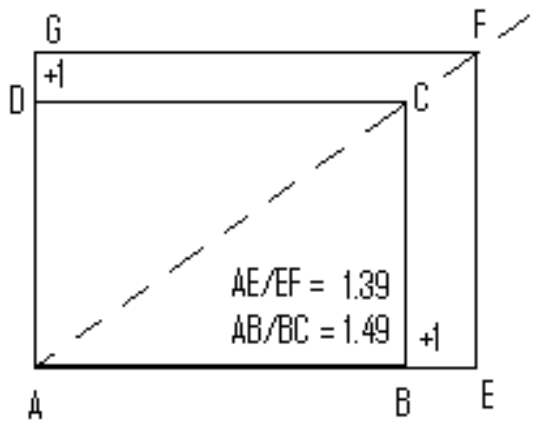

Figura 9

Otro método interesante que emplearon los 29 alumnos del grupo de sexto grado para encontrar un rectángulo proporcional a uno ya dado fue usar el centro y las diagonales de este último (ver Figura 10), los 29 estudiantes determinaron su nuevo rectángulo con el mismo centro y obtuvieron diagonales que coincidieron con las diagonales extendidas del primero, los alumnos se fijaron que como ambos rectángulos eran similares, las medidas de sus lados eran proporcionales.

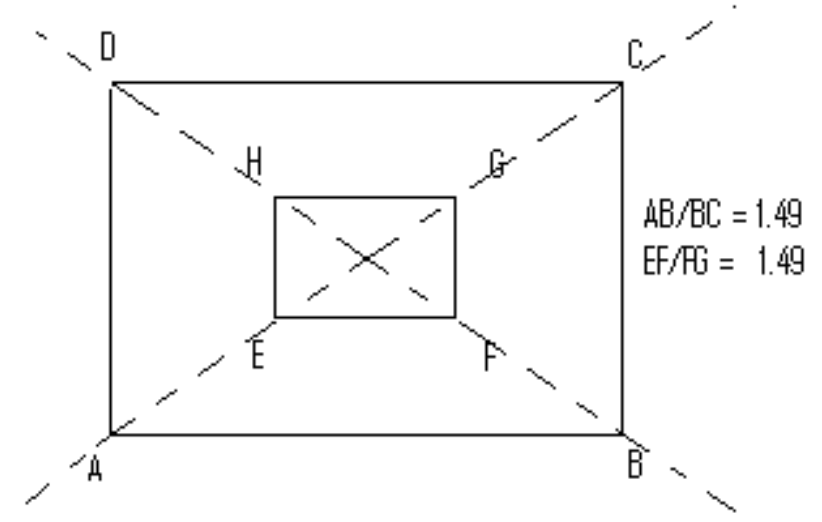

Figura 10. Construcción de rectángulo proporcional

Actividad 2: Construcción de una cancha de fútbol

En primer lugar, los estudiantes trazaron un rectángulo con su largo libre y su ancho en la proporción indicada antes. Este rectángulo jugó el papel del campo de fútbol. Una vez que trazaron el rectángulo, procedieron a trazar las áreas y demás objetos, creando las razones apoyadas el dibujo o bien con el uso combinado de los comandos calculadora y transfe- 
rencia de medidas. Después los estudiantes procedieron dibujando primero todo lo que hacía falta para una mitad del campo y después reflejando todos los objetos usando la línea de medio campo.

\section{c) Análisis de la Actividad 2}

La manipulación directa del largo del campo permitió cambiar de problema a conveniencia de la situación que se desee plantear a los alumnos. Esta es una ventaja de emplear el programa dinámico de geometría en actividades de razón y proporción, además el arrastre de un punto permitió construir rectángulos o en general figuras proporcionales entre sí.

Finalmente, los 29 alumnos del grupo de sexto grado lograron construir canchas proporcionales a la dada (ver figura 11).

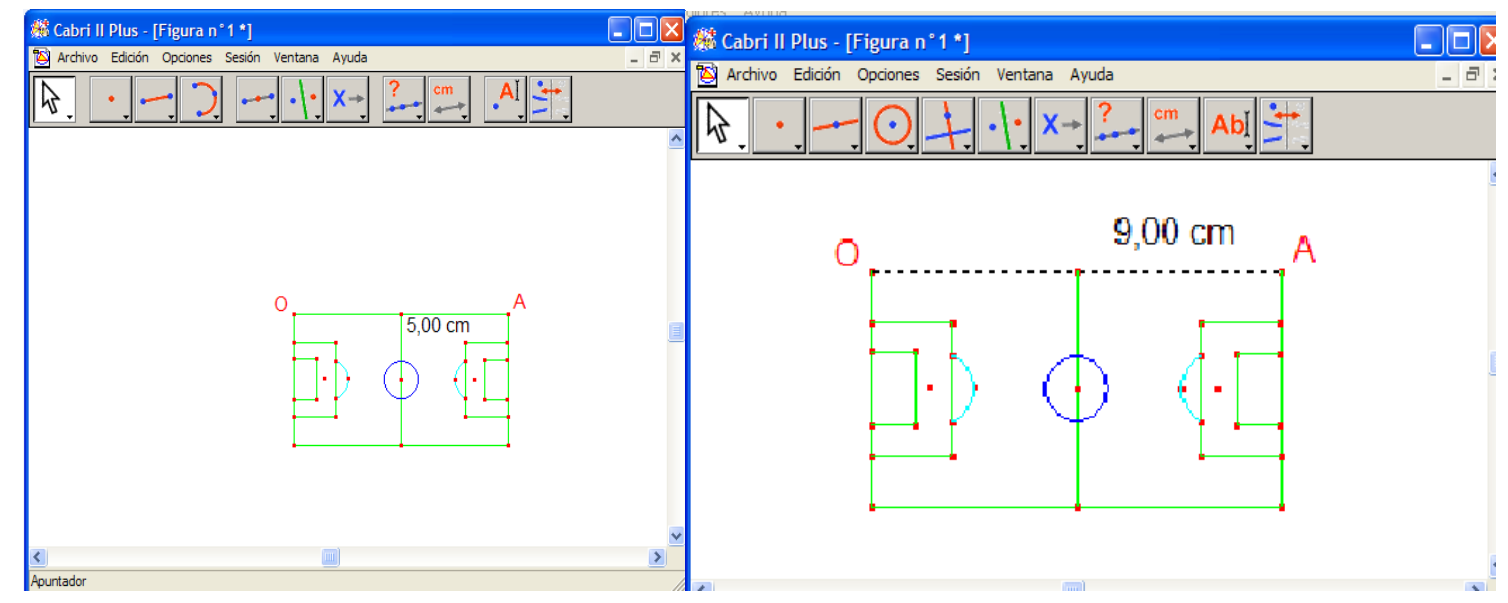

Figura 11. Construcción de la cancha de fútbol

Actividad 3: La fotografia de tu equipo

Los estudiantes emplearon el dibujo para representar las dos estaturas de uno de los integrantes del equipo; la estatura real y la de la foto y en otro segmento colocaron el valor de la estatura de la fotografía de otro miembro del equipo, de esta forma construyeron figuras semejantes.

d) Análisis de la Actividad 3 
Para encontrar la estatura real de la otra persona trabajaron con razones externas y establecieron la proporción correspondiente.

El valor desconocido en este caso era la estatura real de la segunda persona, y para determinarlo emplearon operaciones dividiendo la estatura real de la persona 1 entre la estatura de la foto de la misma persona. El valor obtenido fue dividido entre la estatura de la foto de la segunda persona.

\section{Discusión}

La resolución de estas actividades muestra que los estudiantes, en su mayoría, pudieron determinar las razones involucradas en las figuras y establecer las relaciones de equivalencia que los condujeron a resolver con éxito dichas actividades. Muchos estudiantes corroboraron sus respuestas apoyándose en lo visual, lo que implica que lo cualitativo no fue abandonado totalmente, más bien les permitió a los escolares corroborar su pensamiento proporcional cuantitativo, como lo señala Kieren (1985).

Se pudo verificar que la utilización de la conservación de la proporción en la construcción de micro mundos se usó en el desarrollo de las actividades didácticas, con los tres enfoques señalados en los aspectos teóricos: i) En el caso de emplearse como verificador de los cálculos que hicieron se notó cuando determinaron las canchas proporcionales estableciendo razones de forma numérica, al dar valor a los lados de los rectángulos obtenidos, ii) como generador de nuevos problemas por medio del arrastre de algún punto libre conveniente, se observó cuando los escolares arrastraban un vértice del rectángulo que ejemplificaba la cancha de futbol para hacerla proporcional a la dada o cuando determinaron la estatura de la persona empleando líneas que trazaron de forma vertical y horizontal, revisando que estuvieran en la misma razón y iii) como ilustrador de la transición entre las configuraciones posibles. Lo que se observó cuando manipularon los puntos que les permitió preservar la razón entre altura real y altura en la foto.Se corroboró lo señalado por Franco, (2008) cuando los estudiantes lograron trabajar en los tres registros de representación (el dibujo, la tabla o los datos numéricos), lo que implica que dieron sentido y comprendieron las situaciones planteadas. 
En cuanto al aprendizaje que lograron tener los estudiantes a través del empleo de actividades con lápiz y papel como con del uso del programa de geometría dinámica se puede decir que hubo una conjunción al trabajar en ambos ambientes lo que se muestra cuando empleaban la acción de sobreponer figuras de forma física como arrastrándolas empleando el ratón. Esto indica que comparaban los lados y establecían razones de forma implícita, tal y como lo señala Freudenthal (1983).

Otra acción realizada por los escolares en ambos ambientes fue la de obtener mitades o terceras partes o el doble y determinar así razones de forma numérica y su equivalencia.Los tres registros de representación: dibujo, tabla y uso de datos numéricos fueron empleados en ambos ambientes como fue mencionado en los resultados.

Por otra parte, se considera que para proponer modelos experimentales sólidos que puedan realizarse lo mismo en ambientes de lápiz y papel, como con tecnología se requiere de un marco teórico amplio y que haya sido probado en diversas investigaciones. La experimentación con tecnología de los modelos propuestos aportarán datos para comprender los problemas que tienen los alumnos en torno a las nociones de razón y proporción, en especial su maduración en el transcurso de su educación. Cabe recalcar que las últimas propuestas giran en torno a destacar lo geométrico, que frecuentemente está opacado por el énfasis dado a los aspectos algorítmicos.

Se espera que las nociones primarias adquiridas por el sujeto al respecto de razón y proporción, tengan una evolución al avanzar en la currícula; estudiar las explicaciones y construcciones mentales de los alumnos hace necesario contar con instrumentos que identifiquen y contrasten la madurez de estas nociones.

Hay diferentes actividades para enriquecer y expander el conocimiento de los estudiantes acerca las nociones de razón y proporción. La representación y verificación visual del teorema de Thales, el estudio de funciones lineales, y la computación y representación gráfica de constantes como $\pi$ o la razón dorada puede ser usada para estos propósitos. Adicionalmente, algunas de estas actividades pueden servir como un potente elemento de motivación. 


\section{Referencias}

English, L. (2009). Setting an agenda for international research in mathematics education. En L. English (Ed.), Handbook of international research in mathematics education (pp. 319). New York: Routledge.

Franco, C. (2008). Relajación creative, creatividad motriz y autoconcepto en unamuestra de niños de Educación Infantil. Electronic Journal of Research in Educational Psychology, $14,6(1), 29-50$.

Freudenthal, H. (1983). Didactical Phenomenology of Mathematical Structures. Dordrechet: Reidel.

Guerrero, E., Gil, N. y Blanco, L. (2006). El dominio afectivo en el aprendizaje de las matemáticas. Electronic Journal of Research in Educational Psychology, 8, 4(1), 47-72.

Hart, K. (1988). Ratio and proportion. En J. Hiebert y M. Behr (Eds.), Concepts and operations in the Middle Grades, 2 (198-219). Reston, VA: National Council of Teachers of Mathematics.

Kieren, T., Nelson, D. y Smith, G. (1985). Graphical Algorithms in Partitioning Tasks. The journal of mathematical behavior 4, 25-36.

Lesh, R., Post, T. y Behr., M. (1988). Proportional reasoning. En J. Hiebert y M. Behr (Eds.), Concepts and operations in the Middle Grades, 2 (pp. 93-139). Reston, VA: National Council of Teachers of Mathematics.

Lupiáñez, J. L. y Moreno, L. (2001). Tecnología y Representaciones Semióticas en el Aprendizaje de las Matemáticas. En L. Rico y P. Gómez (Eds.), Iniciación a la Investigación en Didáctica de la Matemática (pp. 291-300). Granada: Editorial Universidad de Granada.

Piaget, J. (1978). Psicología del niño. Madrid: Morata.

Piaget, J. e Inhelder, B. (1978). Las operaciones intelectuales y su desarrollo. En J. Delval (Comp.), Lecturas en Psicología del Niño I (pp. 70-119). Madrid: Alianza Editorial.

Ruiz, E. F. (2002). Estudio de Estrategias de Solución y una Propuesta para la Enseñanza de razón y proporción. Tesis Doctoral. Cinvestav, IPN. México.

Ruiz, E. F. y Valdemoros, M. (2002). Concepts of ratio and proportion in basic level students: case study. En D. Mewborn, P. Sztajn, E. White,H. Wiegel, R. Bryant y K. Nooney (Eds.), Proceedings of the $24^{\text {th }}$ Annual Meeting of the North American Chapter of the International Group for the Psychology of Mathematics Education, Vol. 4 (pp. 16511657). Columbus, OH: Eric Clearinghouse for Science, Mathematics, and Environmental Education. 
Ruiz, E. F. y Valdemoros, M. (2004). Connections between qualitative and quantitative Thinking about proportion: The case of Paulina. En M. J. Hoines y A. B. Flugestad (Eds), Proceedings of the $28^{\text {th }}$ Conference of the International Group for the Psychology of Mathematics Education. Vol. 1 (pp. 201-208). Bergen: Bergen University College.

Santos, L. M. y Espinosa, H. (2002). Searching and exploring properties of geometric configurations using dynamic software. International Journal of Mathematics Education, Science and Techonology, 33(1), 37-50.

Secretaría de Educación Pública (2001a). Plan de programas de estudio. Educación Básica. Primaria. Dirección General de Materiales y Métodos Educativos de la Subsecretaría de Educación Básica y Normal. México D. F.: SEP.

Secretaría de Educación Pública. (2001b). Matemáticas. Sexto grado. México D. F.: Autor.

Streefland, L. (1984). Search for the roots of ratio: Some tough on the long term learning process. Part I. Educational Studies in Mathematics, 15(3), 327-348.

Streefland, L. (1990). Free Productions in Teaching and Learning Mathematics En: K. Gravemeijer, M. Van de Hunal y L. Streefland (Eds.), Contexts Free Productions Tests and Geometry in Realistic Mathematics Education (pp. 33-52). Utrecht: Researchgroup for Mathematical Educational Computer Canter State Univesity of Utrecht.

Streefland, L. (1991). Fractions in realistic mathematics education. Thesis doctoral. Dordrecht: Kluwer Academic Publishers.

Streefland, L. (1993). The design of a mathematics course. A theoretical reflection. Educational Studies in Mathematics, 25(1-2), 109-135. 
[Página en blanco por razones de paginación] 